\title{
Perceptual and Motor Performances between Fencers and Non-Fencers
}

\author{
Selcuk Akpinar ${ }^{1}$ and Ozkan Beyaz ${ }^{1}$ \\ 'Nevsehir Haci Bektas Veli University, Faculty of Education, Department of Physical Education and Sport, Nevsehir, Turkey
}

\begin{abstract}
Sports participation can improve many cognitive and physical performances. It is important to test different sports in different perceptual and motor tests in order to expose the demands of sports. Thus, in this study, we investigated if there are some perceptual and motor performance differences between fencers and aged match sedentary group. Nineteen fencers and nineteen non-fencers performed choice reaction time in an aiming task. Reaction time (RT), accuracy, and velocity of the movements were measured. Results displayed that fencers had significantly faster RT, better accuracy with faster movements compared to non-fencers. These findings suggest that participating a sport requiring fast and accurate pointing movements can modify perceptual and motor performance parameters. Thus, it could be better to select participants who have better perceptual and motor performances. Moreover, speed-accuracy trade off proposed by Fitts (1954) can be altered through long-term sport participation.
\end{abstract}

Key words: accuracy, speed, trade-off, motor performance, reaction time

\section{Introduction}

Participating physical activities and exercise can improve the physical, perceptual, and cognitive well-being (Chan, Wong, Liu, Yu, \& Yan, 2011; Hassmén, Koivula, \& Uutela, 2000; Lee, Hsieh, \& Paffenbarger, 1995). Overall, it has been generally accepted that athletes have better performance in some motor tasks, like balance (Davlin, 2004), strength (Sleivert, Backus, \& Wenger, 1995), and speed than non-athletes. Moreover, superior performance of athletes with the result of the long-term practice has also been observed in some perceptual motor skills, like reaction time (Chan et. al., 2011; Di Russo, Taddei, Apnile, \& Spinelli, 2006). Superior performance of any athletes can be linked to some neurophysiological characteristics. Neurophysiological characteristics help determine superior athletes' level of performance in that athletes' brains must adapt their behaviors to perform skilled movements under different and changing environment (Nakata, Yoshie, Miura, \& Kudo, 2010). Such neural activities in the brain include perception, decision-making, motor preparation, and execution of the movement. Several studies reported changes and shifts in brain activity due to long-term practice among both musicians (Ridding, Brouwer, \& Nordstrom, 2000) and athletes (Pearce, Thickbroom, Byrnes, \& Mastaglia, 2000). Thus, the brain shows great plasticity in its ability to acquire skills over the long-term, and in turn to improve performance when executing skilled movement.

Besides the perceptual motor skills, athletes also displayed better sensorimotor performance in comparison to non-athletes both their dominant and non-dominant arms in many sports (Akpinar \& Bicer, 2014; Akpinar, 2015: Akpinar; 2016: Akpinar, Sainburg, Kirazci, \& Przybyla, 2015; Ramsay \& Riddoch, 2001). The effect of long-term practice on sensorimotor performance has also been observed in musicians (Rodrigues, Loureiro, \& Caramelli, 2013). The observed improvements in physical and perceptual functioning are somehow associated with the length of sport participation (Brisswalter, Collardeau, \& René, 2002). Therefore, the improvements can be seen with the long-term participation.

One of the sports that requires a high level of perceptual and motor performance is fencing. Fencing is a combat sport

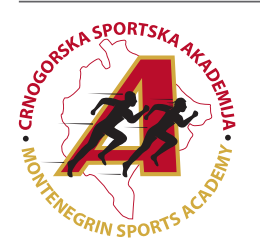

Correspondence:

Nevsehir Haci Bektas Veli University, Faculty of Education, Department of Physical Education and Sport, 50300, Nevsehir, Turkey E-mail: selcukbos@gmail.com 
in which two athletes fight indirectly, through their weapons (the foil, the sabre, or the epee), and mainly includes fast movements directed toward the opponent. Those movements need to be fast and accurate so that the fencer can get a point. Regarding the speed and accuracy, Fitts (1954) stated that when the speed of the movement increased then this reduces the accuracy of movement. In opposite, decreasing the speed of the movement increase the accuracy of the movement. This phenomenon, speed accuracy trade-off, can be often seen in many movements. It is still crucial to state that nature of the task can determine how this phenomenon affects the performance. When the task is mainly a fine motor skill or when it is a gross motor skill, then the effect of speed accuracy tradeoff may be different in those skills. Some skills require both to be accurate and fast to accomplish the task (i.e. batting in baseball). In these cases, studies suggest that moving with an optimal speed will yield an accurate movement performance (Belkin \& Eliot, 1997; Freeston \& Rooney, 2014).

Years of training improve coordination of both arms of right-handed fencers, making them less lateralized than are non-athletes (Akpinar et al., 2015). Fencing was chosen in this study because it challenges upper limb coordination. As it was stated above, sports participation can increase the well being of physical, perceptual, and cognitive parameters, we tried to investigate if the fencers have better perceptual and motor performance in an aiming task compared to non-fencers. Moreover, we wanted to figure out if fencers will display different pattern in speed accuracy trade-off compared to non-fencers. The reason to ask this question is basically fencers make fast pointing movements and those movements also need to be accurate. Thus, they may show different pattern in terms of speed accuracy compared to non-fencers.

\section{Method}

Nineteen fencers (10 female) between 19 and 24 years old $\left(\mathrm{M}_{\mathrm{age}}=22.8 \pm 2.45\right)$ and nineteen healthy young non-fencers $(10$ female) between 18 and 25 years old $(21.3 \pm 2.12)$ signed voluntarily informed consent form approved by Nevsehir Haci Bektas Veli University which was conducted in accordance with the Declaration of Helsinki as amended by the World Medical Association Declaration of Helsinki (World Medical Association, 2013). Fencers' experiences changed between 4 and 9 years and non-fencers self reported no training experience in any sports. All the participants reported right handedness and scored above $60 \%$ on the extended 35 -item handedness questionnaire (Hull, 1936), which is similar to widely known Edinburgh Inventory (Oldfield, 1971).

\section{Experimental Setup}

The detail of the experimental setup was previously explained (Akpinar, 2016). The participants seated an adjustable chair with a sensor of the electromagnetic movement tracker (TrackSTAR, Ascension Technology, USA) put to their right forearm. 2D view for reaching was provided to the participants. There was a mirror above the participants' arms and this mirror displayed one cursor, one start circle for each hand, and 3 different targets that were projected from 55" flat TV. This configuration was designed from a custom virtual reality interface. This cursor was associated with the index finger of each arm and its position on the mirror was updated in real time that was limited to TV screen update of $100 \mathrm{~Hz}$.

There were three targets in different directions $\left(80^{\circ}, 90^{\circ}\right.$, and $100^{\circ}$, please see Figure 1) and the task was to reach these targets with maintaining accuracy. One target was shown for each trial randomly. The start circle was $2 \mathrm{~cm}$ in diameter and was $20 \mathrm{~cm}$ away from the body midline to the right side. Each target was shown as $3.5 \mathrm{~cm}$ in diameter. There was a cursor representing the tip of the index finger. The cursor was $1 \mathrm{~cm}$ in diameter with cross hair. The distance between the start circle and target was set to $30 \mathrm{~cm}$ so that each participant could reach the target easily. We gave an imperative audio-visual "go" signal, upon which participants were required to move to the target. The imperative signals were delayed until the participants positioned their right hand in start location for 300 milliseconds. The task was to move the cursor to the target, which was displayed when the cursor placed in the start circle. Thus it was restricted pace, that is, the participants did not know which target would pop-up in the screen.

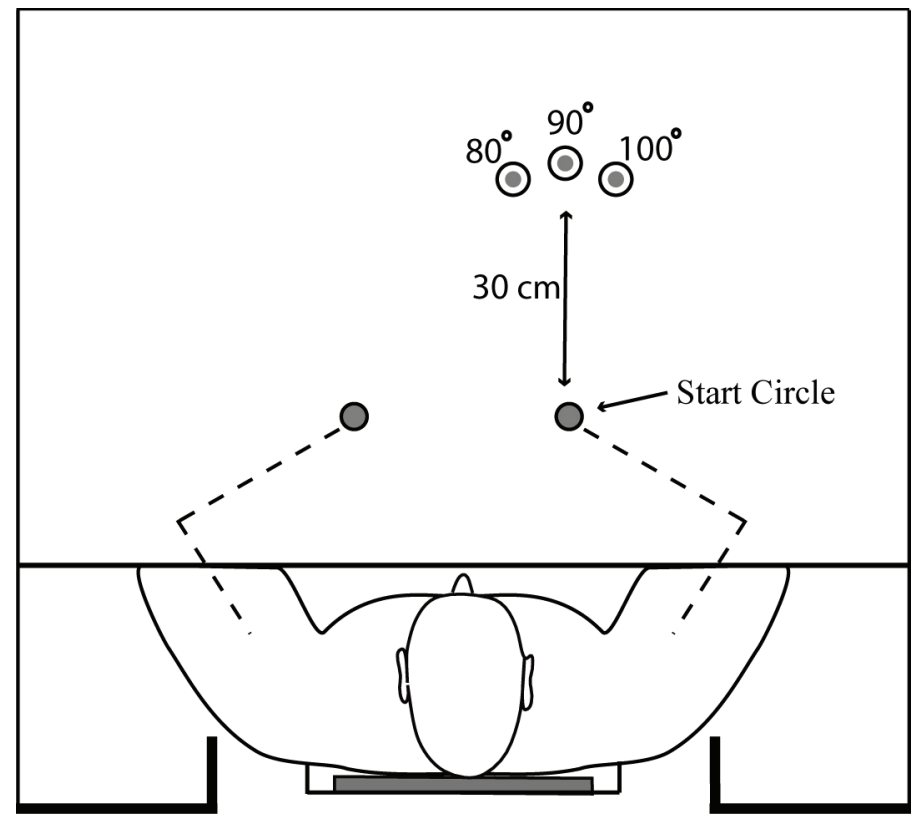

Figure 1. The distribution of the targets 


\section{Experimental Task}

As it was stated above, there were three different targets and participants performed the total of 30 trials ( 10 trials to each target). Participants were asked to make fast reaches to the targets (trials were $1 \mathrm{sec}$ ) while maintaining accuracy with possibly no additional corrections. In order to motivate the participants, accuracy of the trials were rewarded with 10,3 and 1 point for landing within $3.5 \mathrm{~cm}, 4.5 \mathrm{~cm}$ and $5.5 \mathrm{~cm}$ diameter from the center of the target respectively. After each trial, feedback about the score and performed cursor path were displayed for $1 \mathrm{sec}$. The task condition can be considered as choice reaction time condition because there was more than one stimulus. Experimental task only performed with the right dominant arm by the participants.

\section{Data and Statistical Analysis}

In order to determine perceptual and movement performance, we quantified three measures: 1) The reaction time (RT) was defined as the elapsed time between the presentation of a target on the workplace and the initiation of the movement to that target; 2) Movement accuracy (Final Position Error=FPE); 3) Movement speed was defined as the peak amplitude of the velocity profile. The collected data were analyzed using Matlab software and, dependent variables were calculated.

For the statistical comparison, the mean score of each dependent measures was calculated and subjected to the statistical analysis. For the statistical comparison, t-test was used and statistical significance level was set as .05.

\section{Results}

Both groups, fencers and non-fencers, made reaches to the three different targets located across horizontal space in front of the body with the dominant arm. Figure 2 shows the average magnitude of the reaction time (RT).The statistical analysis displayed a significant result for RT, $\mathrm{t}_{(36)}=3.35$, $\mathrm{p}=.001$.Fencers' RT was $(\mathrm{M}=273 \pm 42 \mathrm{~ms})$ faster compared to non-fencers $(\mathrm{M}=327 \pm 56 \mathrm{~ms})$.

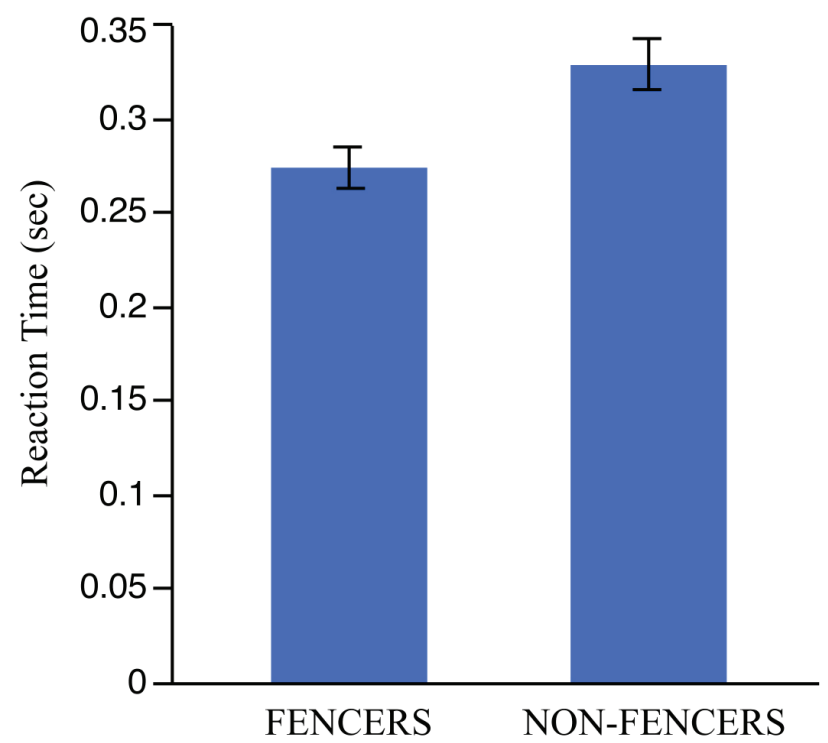

Figure 2. The average magnitude of the reaction time (RT) between fencers and non-fencers

The other dependent variable was final position error (FPE). The mean value for FPE for both groups was displayed in Figure 3. The statistical analysis showed a significant re- sult for FPE, $\mathrm{t}_{(36)}=14.93, \mathrm{p}=.0001$. Fencers had significantly less errors $(\mathrm{M}=3.79 \pm 1.74 \mathrm{~cm})$ compared to non-fencers $(\mathrm{M}=23.04 \pm 5.33 \mathrm{~cm})$.

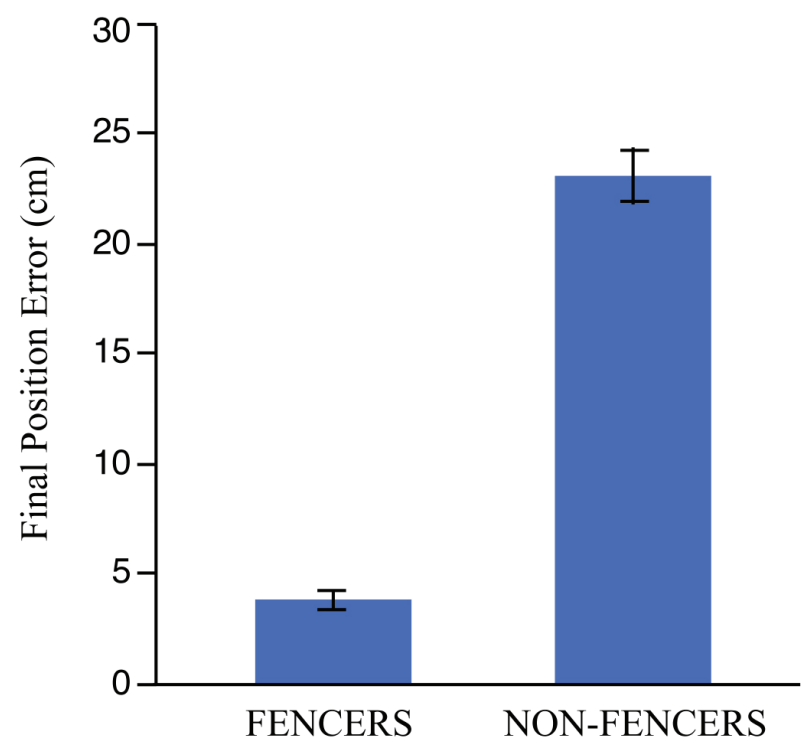

Figure 3. The average magnitude of final position error (FPE) between fencers and non-fencers 
We have lastly measured movement speed during the reaches. The mean value for movement speed for both groups was given in Figure 4. The statistical analysis showed a signif- icant result for movement speed, $\mathrm{t}_{(36)}=-7.49, \mathrm{p}=.0001$. Fencers' reaches were significantly faster $(\mathrm{M}=183.6 \pm 22.3 \mathrm{~cm})$ compared to non-fencers $(\mathrm{M}=124.5 \pm 26.1 \mathrm{~cm} / \mathrm{s})$.

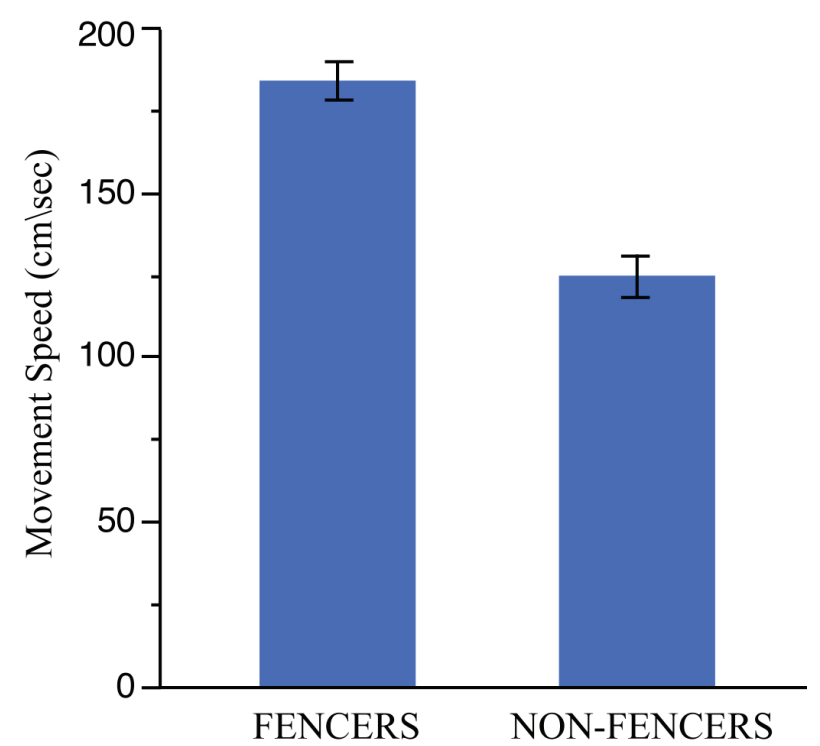

Figure 4. The average magnitude of movement speed between fencers and non-fencers

\section{Discussion}

In study, we examined perceptual and motor performances between fencers and non-fencers during a reaching task with a choice reaction time (RT) condition. The variables depicting the perceptual (RT) and motor performances (accuracy and movement speed) were analyzed. In all those variables, fencers displayed better performances compared to non-fencers. That is, fencers performed the reaching task with better RT, less final position error, and faster movements compared to non-fencers.

Superior performance of fencers compared to non-fencers was observed in many tasks. For instance, greater performance of the right arm in fencers over non-fencers has been reported for discriminative reaction time tasks (Chan et al., 2011; Di Russoet al., 2006). In their study, Chan et al. (2011) concluded that the combination of physical fitness and level of expertise get more benefit for cognitive control mechanism compared to when each of them applied singly. In the current study, we also found that fencers have better perceptual performance compared to non-fencers. Both cognitive and perceptual skills are acquired through training, and the long-term participation of training required to attain high level of skill makes the component processes mainly automatic (Logan, 1988). Thus, expert skills are often flexible, so they can be utilized in various task contexts (MacKay, 1982), like what was observed in the current study. In another study, Williams and Walmsley (2000) introduced recordings of EMG activity during measurement of response times between elite fencers and novice subjects. They have found that elite fencers displayed more coherent muscle synergies and more consistent pattern of muscle coordination than novice subjects. Thus, more coherent muscle synergies for fencers may lead to have fewer errors than non-fencers, which was observed in the current study. We have also found that fencers' reaches were significantly faster than non-fencers. It has been previously found that fencers were faster than non-fencers in movements of the upper limbs (Roi \& Bianchedi, 2008); thus, our finding on reaching movement speed is in agreement with that previous study re- sult. Participation of long-term practice may lead the fencers to develop this skill over the time. In addition, as they mainly practice fast pointing movements in their exercise settings, this can lead them to have faster movements than non-fencers. In fact these results are not in agreement with speed accuracy trade-off proposed by Fitts (1954). As we stated earlier, Fitts stated that when the movement velocity increased, the errors in the aiming movements increased as well. This trend was not observed for the fencer. The speed accuracy trade-off has been an interesting topic for researchers especially focusing on choice RT tasks (Bogac, Wagenmakers, Forstmann, \& Nieuwenhuis, 2010). This phenomenon is also very important for the sports performance. For instance, Freeston and Rooney (2014) conducted a study with baseball and cricket players to determine the speed that optimizes accuracy in a throwing task. They found that speed accuracy trade-off was worse for the cricket players compared to baseball players. Thus, even though these two sports seem to be similar in many ways, they do not show the similar pattern in motor performance. In our study, fencers showed faster reaches with significantly less errors compared to non-fencers. Thus, speed accuracy trade-off is not predetermined entity and can be modified by long-term sport participation. It has been also previously stated that the classical Fitts' law can be violated in tasks that involve a ballistic component (Juras, Slomka, \& Latash, 2009). In this study, we compared fencers and non-fencers in the same task that can include a ballistic component, and fencers showed a different pattern compared to non-fencers and violated the speed accuracy trade-off. In conclusion, although we do not know if the fencers had already superior perceptual and motor skill capabilities before they started fencing, we can point that this sport requires high perceptual and motor skill requirement. Moreover, fencers can control better the speed accuracy trade-off, and thus the phenomenon cannot be valid for some groups.

\section{Acknowledgements}

There are no acknowledgements. 


\section{Conflict of Interest}

The authors declare that there are no conflicts of interest.

Received: 16 August 2018| Accepted: 19 September 2018| Published: 01 October 2018

\section{References}

Akpinar, S. (2015). The effect of long-term bimanual training on arm selection during reaching tasks. Kinesiology, 47(2), 226-235.

Akpinar, S. (2016). Decreased interlimb differences in female basketball players. The Journal of Sports Medicine and Physical Fitness, 56(12), 1448-54.

Akpinar, S. \& Bicer, B. (2014). Why Left-Handers/Footers Are Overrepresented in Some Sports. Montenegrin Journal of Sports Science and Medicine 3(2), 33-38.

Akpinar, S., Sainburg, R. L., Kirazci, S., \& Przybyla, A. (2015). Motor Asymmetry in Elite Fencers. Journal of Motor Behavior, 47(4), 302-11.

Belkin, D.S., \& Eliot, J.F. (1997). Motor skill acquisition and the speed accuracy tradeoff in a field based task. Journal of Motor Behavior, 47, 144-152.

Bogac, R., Wagenmakers, E.J., Forstmann, B.U., \& Nieuwenhuis, S. (2010). The neural basis of the speed-accuracy tradeoff. Trends Neurosci, 33, 10-16.

Brisswalter, J., Collardeau, M., \& René, A. (2002) Effects of acute physical exercise characteristics on cognitive performance. Sports Med, 32, 555-566. doi: 10.2165/00007256-200232090-00002.

Chan, J.S.Y., Wong, A.C.N., Liu, Y., Yu, J., \& Yan, J.H. (2011). Fencing expertise and physical fitness enhance action inhibition. Psychology of Sport and Exercise, 12, 509-514.

Davlin, C.D. (2004). Dynamic balance in high level athletes. Percept Mot Skills, 98, 1171-1176.

Di Russo, F., Taddei, F., Apnile, T., \& Spinelli, D. (2006). Neural correlates of fast stimulus discrimination and response selection in top-level fencers. Neurosci Lett, 408(2), 113-118.

Fitts, P.M. (1954). The information capacity of the human motor system in controlling the amplitude of movement. Journal of Experimental Psychology, 47, 381-391.

Freeston, J. \& Rooney, K. (2014). Throwing Speed and Accuracy in Baseball and Cricket Players. Perceptual and Motor Skills, 118(3), 637-650.

Hassmén, P., Koivula, N., \& Uutela, A. (2000). Physical exercise and psychological well-being: a population study in Finland. Prev Med, 30(1), 17-25.
Hull, C. (1936). A study of laterality test items. J Exp Educ, 4, 287-290.

Juras, G., Slomka, K., \& Latash, M. (2009). Violations of Fitts' Law in a Ballistic Task. Journal of Motor Behavior, 41(6), 525-528.

Lee, I.M., Hsieh, C.C., \& Paffenbarger, R.S. (1995). Exercise intensity and longevity in men. The Harvard Alumni Health Study. JAMA, 273, 1179-1184.

Logan, G.D. (1988). Toward an instance theory of automatization. Psychological Review, 95, 492-527. doi:10.1037/0033-295X.95.4.492

MacKay, D.G. (1982). The problem of flexibility, fluency, and speed-accuracy tradeoff in skilled behavior. Psychological Review, 89, 483-506.

Nakata, H., Yoshie, M., Miura, A., \& Kudo, K. (2010). Characteristics of the athletes' brain: Evidence from neurophysiology and neuroimaging. Brain Research Reviews, 62(2), 197-211.

Oldfield, R.C. (1971). The assessment and analysis of handedness: the Edinburgh inventory. Neuropsychologia, 9(1), 97-113.

Pearce, A.J., Thickbroom, G.W., Byrnes, M.L., \& Mastaglia, F.L. (2000). Functional reorganisation of the corticomotor projection to the hand in skilled racquet players. Exp Brain Res, 130(2), 238-243.

Ramsay, J.R., \& Riddoch, M.J. (2001). Position-matching in the upper limb: professional ballet dancers perform with outstanding accuracy. Clin Rehabil, 15(3), 324-330.

Ridding, M.C, Brouwer, B., \& Nordstrom, M.A. (2000). Reduced interhemispheric inhibition in musicians. Exp Brain Res, 133(2), 249-253.

Rodrigues, A.C., Loureiro, M.A., \& Caramelli, P. (2013). Long-term musica training may improve different forms of visual attention ability. Brain Cogn, 82(3), 229-235.

Roi, G.S. \& Bianchedi, D. (2008). The science of fencing: implications for performanceand injury prevention. Sports Medicine, 38, 465-481.

Sleivert, G.G., Backus, R.D., \& Wenger, H.A. (1995). Neuromuscular differences between volleyball players, middle distance runners and untrained controls. Int J Sports Med, 16(6), 390-398.

Williams, L.R. \& Walmsley, A.(2000). Response timing and muscular coordination in fencing: a comparison of elite and novice fencers. J Sci Med Sport, 3, 460-475.

World Medical Association. (2013). World Medical Association Declaration of Helsinki: Ethical Principles for Medical Research Involving Human Subjects. The Journal of the American Medical Association, 310(20), 21912194. 